BMJ Open

Diabetes

Research

\& Care

\title{
Risk of morbidity and mortality in patients with type 2 diabetes treated with sodium-glucose cotransporter-2 inhibitor and/or dipeptidyl peptidase-4 inhibitor: a nationwide study
}

\author{
Gábor Sütő, ${ }^{1}$ Gergő A Molnár (10 , , Gyorgy Rokszin, ${ }^{2}$ Ibolya Fábián, ${ }^{2}$ Zoltan Kiss, ${ }^{1}$ \\ Zoltán Szekanecz, ${ }^{3}$ Gyula Poór, ${ }^{4}$ György Jermendy, ${ }^{5}$ Peter Kempler, ${ }^{6}$ \\ István Wittmann (iD ${ }^{1}$
}

\begin{abstract}
To cite: Sütő G, Molnár GA Rokszin G, et al. Risk of morbidity and mortality in patients with type 2 diabetes treated with sodium-glucose cotransporter-2 inhibitor and/ or dipeptidyl peptidase-4 inhibitor: a nationwide study. BMJ Open Diab Res Care 2021;9:e001765. doi:10.1136/ bmjdrc-2020-001765
\end{abstract}

- Supplemental material is published online only. To view please visit the journal online (http://dx.doi.org/10.1136/ bmjdrc-2020-001765).

Received 9 July 2020 Revised 12 December 2020 Accepted 30 December 2020

Check for updates

(c) Author(s) (or their employer(s)) 2021. Re-use permitted under CC BY-NC. No commercial re-use. See rights and permissions. Published by BMJ.

For numbered affiliations see end of article.

Correspondence to Professor István Wittmann; istvan.wittmann@aok.pte.hu

\section{ABSTRACT}

Introduction Mortality and disability in diabetes mellitus are determined mostly by cardiovascular complications and cancer. The impact of dipeptidyl peptidase- 4 inhibitor (DPP-4i) and sodium-glucose cotransporter-2 inhibitor (SGLT2i) monotherapy or combination on long-term complications of type 2 diabetes mellitus was studied. Research design and methods Patients with type 2 diabetes treated with DPP-4i or SGLT2i during a 3-year period were identified in the database of the National Institute of Health Insurance Fund in Hungary. All-cause mortality, acute myocardial infarction, stroke, hospitalization for heart failure (HHF), lower limb amputation (LLA) and cancer were assessed. Outcomes of add-on SGLT2i to DPP-4i treatment in comparison with switching DPP-4i therapy to SGLT2i were also evaluated. After propensity score matching, survival analysis was performed with a Cox proportional hazards model. Results After propensity score matching, both SGLT2i and DPP-4i groups included 18583 patients. All-cause mortality (HR, $0.80 ; 95 \% \mathrm{Cl} 0.68$ to $0.94 ; \mathrm{p}=0.0057$ ), HHF ( $\mathrm{HR}, 0.81 ; 95 \% \mathrm{Cl} 0.71$ to $0.92 ; \mathrm{p}=0.0018$ ), and risk of cancer (HR, $0.75 ; 95 \% \mathrm{Cl} 0.66$ to $0.86 ; \mathrm{p}<0.0001)$ were lower in the SGLT2i population compared with DPP-4i. Risk of LLA was higher in the SGLT2i group (HR, 1.35; 95\% Cl 1.03 to $1.77 ; p=0.0315)$. SGLT2i in combination with DPP$4 \mathrm{i}$ results in lower all-cause mortality $(\mathrm{HR}, 0.46 ; 95 \% \mathrm{Cl}$ 0.31 to $0.67 ; p=0.0001$ ), with a lower trend in stroke, LLA, HHF and cancer, but without any statistical difference. Conclusions SGLT2i treatment leads to a lower risk of overall mortality, HHF and cancer when compared with DPP-4i treatment. Adding SGLT2i to DPP-4i instead of switching from DPP-4i to SGLT2i further lowers the risk of all-cause mortality.

\section{INTRODUCTION}

Since acute metabolic complications have become less frequent and less severe, longterm outcomes of patients with type 2 diabetes mellitus (T2DM) have become more and more important. ${ }^{1-3}$ Diabetes mellitus is associated with a twofold to fourfold increase in

\section{Significance of this study}

What is already known about this subject?

- Sodium-glucose cotransporter-2 inhibitor (SGLT2i) has been found to positively influence cardiovascular complications and may reduce cancer death as well.

> Data of patients taking SGLT2i and/or dipeptidyl peptidase-4 inhibitor (DPP-4i) have been analyzed from the records of the National Institute of Health Insurance Fund in Hungary during a 5-year long period.

What are the new findings?

- SGLT2i treatment was found to result in lower allcause mortality, hospitalization for heart failure and risk of cancer and there was a declining trend in the prevalence of stroke in comparison with DPP-4i treatment.

- Adding SGLT2i to DPP-4i results in markedly lower all-cause mortality in comparison with switching from DPP-4i to SGLT2i.

- Adding SGLT2i to DPP-4i instead of switching from DPP-4i to SGLT2i may prove to be a beneficial approach in terms of improving all-cause mortality and neutralizing the effect of SGLT2i on the risk of lower limb amputation.

How might these results change the focus of research or clinical practice?

- The data obtained from the real world enhance our knowledge on switching or combining different treatment modalities for diabetes mellitus.

- The results point to the direction of clinical research on long-term complications influencing morbidity and mortality in diabetes mellitus.

mortality, compared with the general population. $^{4-6}$ Cardiovascular (CV) mortality seems to be a major determinant of all-cause mortality. ${ }^{4} 6$ The most extensively studied 
outcomes are major adverse CV events, most frequently involving non-fatal myocardial infarction, non-fatal stroke and CV death. Recently, especially in the case of sodiumglucose cotransporter-2 inhibitor (SGLT2i), hospitalization for heart failure (HHF) has become another focus of interest. ${ }^{78}$ Initial cardiovascular outcome trials (CVOTs) have demonstrated non-inferiority, that is, safety of drugs. The superiority of empagliflozin (EMPA-REG OUTCOME $)^{7}$ was first proven. Further studies ${ }^{8-10}$ justified benefits with novel agents as well. The benefit of SGLT2i was confirmed by analysis of larger databases or pragmatic, non-randomized (CVD-REAL, CVD-REAL Nordic and EMPRISE) trials providing real-world evidence (RWE). The need for RWE studies is striking, since real-world data do not always coincide with the findings of CVOTs. ${ }^{11}$

CVOT studies have changed the guidelines. For example, the 2018 American Diabetes Association (ADA) and European Association for the Study of Diabetes (EASD) joint position statement recommends that after initial metformin treatment, $\mathrm{CV}$ risk or the presence of heart failure or chronic kidney disease should be taken into account. SGLT2i is recommended for patients with HHF or chronic kidney disease. The consensus was briefly updated in 2019. ${ }^{12}$ Although hemoglobin $\mathrm{A}_{1 \mathrm{c}}\left(\mathrm{HbA}_{1 \mathrm{c}}\right)$ represents a gradual increase in risk, associated with lowest mortality at a range of 6\%-6.9\%, ${ }^{4}$ therapeutic recommendations suggest administration of glucagon-like peptide-1 receptor agonists (GLP-1 RA) and SGLT2i, independent of baseline $\mathrm{HbA}_{1 \mathrm{c}}$ or individualized $\mathrm{HbA}_{1 \mathrm{c}}$ target. ${ }^{12}$ Stroke and lower limb amputation (LLA) are consequences of macrovascular and microvascular complications. Stroke is only mildly influenced by glucose-lowering treatment, while observations on LLAs are conflicting. Some sources did not show any significant alteration, but a substantial increase was also observed. ${ }^{13-15}$

Another leading cause of mortality in patients with type 2 diabetes is cancer. Diabetes itself is associated with a substantially higher risk of different types of cancer. ${ }^{16}{ }^{17}$ Available reports do not allow drawing a conscious conclusion about the relationship of cancer and glucose-lowering compounds.

The recommended initial therapy for T2DM is metformin after diet modifications and lifestyle changes. There are several options to expand medical therapy thereafter. SGLT2i and dipeptidyl peptidase-4 inhibitor (DPP-4i) may be combined with metformin due to different mechanisms; the combination of SGLT2i and DPP-4i with metformin is a desirable option to enhance glucose-lowering effect. The potential effect of the triple combination of metformin, SGLT2i and DPP-4i on complications including mortality and CV risk still awaits confirmation.

The aim of our study was to assess the risk of mortality and morbidity in patients with T2DM treated with SGLT2i or DPP-4i alone or in combination: in one part of the study SGLT2i versus DPP-4i therapy, and in the other part DPP-4i treatment switched to SGLT2i versus SGLT2i added to DPP-4i were compared.

\section{RESEARCH DESIGN AND METHODS}

\section{Data sources and study populations}

A nationwide, retrospective, longitudinal study was performed using the National Institute of Health Insurance Fund (NHIF) database. This is a nationwide insurance system covering close to $100 \%$ of the Hungarian population and collecting patient identification and International Classification of Diseases (ICD)-10 code information on all inpatient and outpatient visits, as well as on all prescriptions of reimbursed drugs including antidiabetic drugs, and the date of death from the mortality database issued by the Central Statistical Office.

All patients in the NHIF database who had taken DPP-4i or SGLT2i between August 1, 2014 and July 31, 2017 were identified (figure 1 and online supplemental figure 1). Patients aged younger than 18 years, women with gestational diabetes (ICD-10: O24.4) and those with polycystic ovary syndrome (ICD-10: E28.2) or those who had dialysis treatment were excluded. Only patients with T2DM were considered in the current analysis, and we excluded those who met the criteria of type 1 diabetes mellitus published earlier (figure 1). ${ }^{18}$

Our study aimed to compare the efficacy of SGLT2i versus DPP-4i therapies (called SGLT2i 'clean' and DPP-4i 'clean' groups), as well as evaluate the outcomes of choice of therapy, switching DPP-4i therapy to SGLT2i treatment versus add-on SGLT2i to DPP-4i (called SGLT2i 'switch' and SGLT2i 'add-on' groups).

First, we created two cohorts: one for those who started DPP-4i therapy and another for SGLT2i users within the index period from August 1, 2014 to July 31, 2017 who did not take these medications 12 months before the index date (figure 1). The index date was defined as the first day of introducing SGLT2i or DPP-4i treatment. Patients who started both SGLT2i and DPP-4i within the index period were excluded. The index period was the same and a 12-month long drug-free period was required for both arms. Previous treatment with DPP-4i and the period from diagnosis to treatment were balanced during propensity score matching (online supplemental table 1). Consequently, the effect of immortal bias for the CVDREAL study, which was criticized, ${ }^{19}$ was prevented. The study period started from August 1, 2014 and closed on July 31, 2019. Patients were followed from the index date until the date of the investigated event, date of death, or end of study period. They were censored in case there was no disposal of the investigated therapy for 90 days after the last prescription of treatment or had a prescription of the drug of the opposite arm.

In the second part of the study, we selected patients who had any DPP-4i therapy 3 months before the first day of SGLT2i prescription (figure 1). The SGLT2i 'switch' arm was identified if patients had no DPP-4i disposal and were still alive after 3 months of SGLT2i index date. We followed patients in this arm until occurrence of the investigated outcome, date of death, or end of study period. Patients were censored in case they did not have 


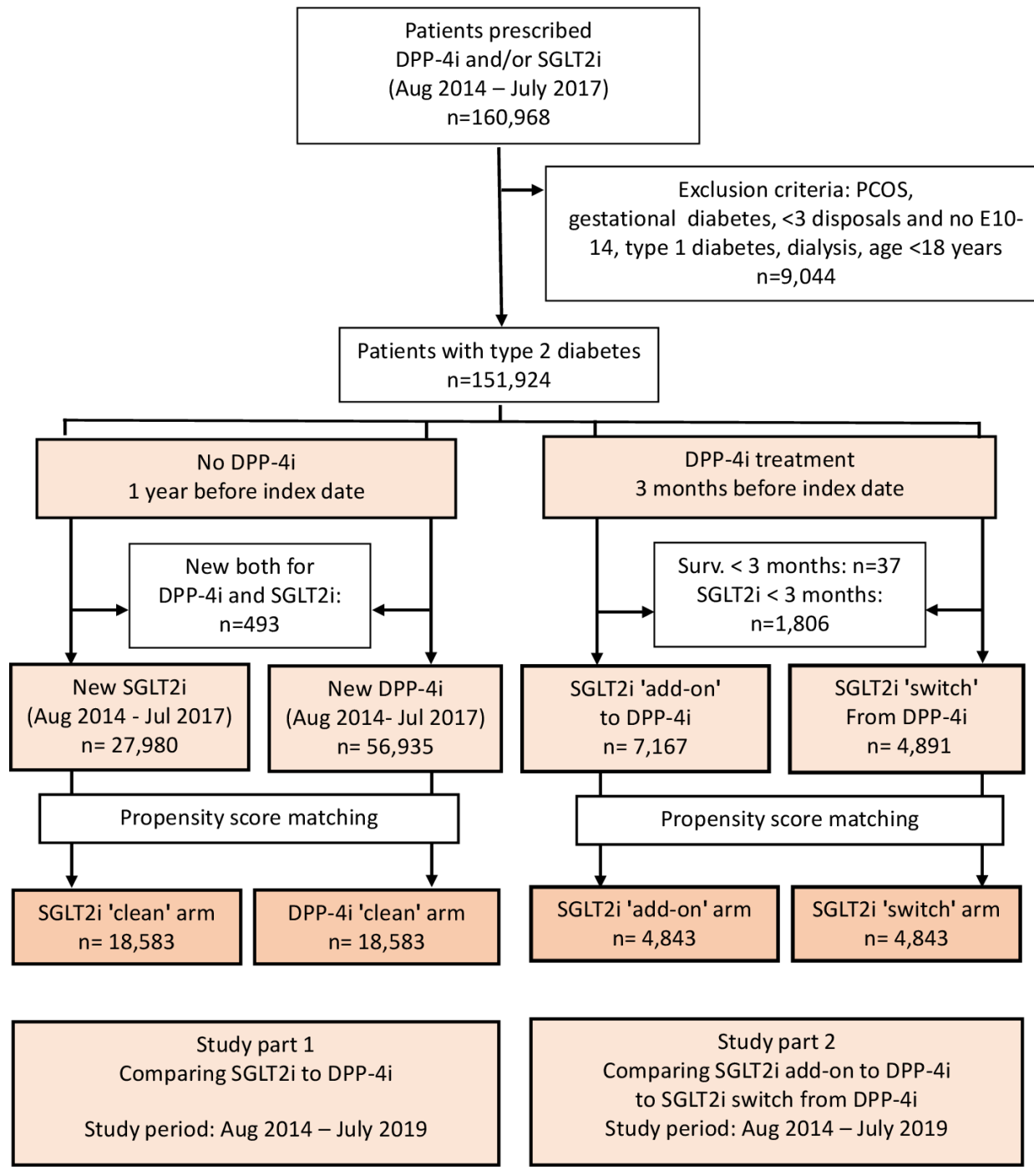

Figure 1 Flow chart of patient enrollment and exclusions for the SGLT2i 'clean' versus DPP-4i 'clean' cohorts, as well as for the SGLT2i 'add-on' versus SGLT2i 'switch' cohorts. The flow chart also indicates the change in the number of cases due to propensity score matching. DPP-4i, dipeptidyl-peptidase-4 inhibitor; PCOS, polycystic ovary syndrome; SGLT2i, sodiumglucose cotransporter-2 inhibitor.

SGLT2i medication for more than 90 days following the last prescription or until the date of any repeated prescription of DPP-4i therapy.

In case of 'add-on' SGLT2i to DPP-4i arm, patients with T2DM had to meet the following requirements: (1) any DPP-4i treatment 3 months before the first day of SGLT2i prescription; (2) in case they were on SGLT2i treatment for at least 3 months and had at least one prescription of DPP-4i therapy within 3 months after the index date; and (3) were alive for at least 3 months after SGLT2i initiation. Patients were followed until the investigated event, date of death or end of study period, or for 90 days after the last disposal.

Patients who were censored in the first ('clean') DPP-4i arm of the study due to addition of SGLT2i to DPP-4i or switching from SGLT2i to DPP-4i were investigated in the second part of the study in case they met the criteria for inclusion.
Baseline patient characteristics including age, sex, index date, date of T2DM diagnosis, prior comorbidities and previous non-antidiabetic medications were described during the screening period from January 1, 2009 to the index date (online supplemental tables 1 and 2; definitions are provided in online supplemental table 3). Previous medication was defined as any drug prescribed in the 12 months prior to the index date.

To prevent the impact of time-lag bias, ${ }^{19}$ we defined the period between the date of diagnosis of T2DM and the index date, and the length of prior DPP-4i treatment, and the ratio of these two periods was adjusted during propensity score matching (online supplemental tables 1 and 2). Aiming to manage time-lag bias, the different types of treatments, including insulin therapy, during the first year of screening period were calculated and balanced during propensity score matching. 
Since the index period of DPP-4i and SGLT2i was the same (starting with the availability date of SGLT2i in Hungary) and a 12-month drug-free period was required for both arms, and with the rate of previous DPP-4 users and the duration of T2DM being balanced during propensity score matching, the effect of immortal bias could also be eliminated.

We investigated the outcomes of all-cause mortality, myocardial infarction together with percutaneous coronary intervention (PCI), diagnosis of ischemic or hemorrhagic stroke confirmed on CT performed 30 days before or after the record date of stroke, and primary or secondary HHF. Further predefined outcomes were LLA and primary or secondary diagnosis of cancer requiring hospitalization.

Definitions of diagnoses and outcomes are detailed in online supplemental table 3 .

\section{Propensity score matching}

Propensity scores were used to match each patient who initiated an SGLT2i with those who started DPP-4i in the 'clean' part at a ratio of 1:1 with a caliper of 0.2. The probability of initiation of an SGLT2i was estimated using a logistic regression model, with all available patient variables at the index date considered as independent variables. Propensity scores were used to match each patient who initiated an SGLT2i 'add-on' arm with patients in the SGLT2i 'switch' arm in the 'add-on' part at a ratio of 1:1 with a caliper of 0.2 . The probability of initiation of an SGLT2i add-on was estimated using a logistic regression model, with all available patient variables at the index date considered as independent variables. Altogether 54 parameters were used for propensity score matching in both parts.

\section{Statistical analysis}

After matching, a standardized difference of more than $10 \%$ was used to detect significant group imbalance between baseline variables. Survival analysis was performed using Cox proportional hazards model, with time since the index date as the underlying timescale. Proportionality assumptions were tested. All matching and analyses were performed with R V.3.6.1 statistical software.

\section{RESULTS}

A total of 84915 patients were included in the analysis. We identified 27980 and 56935 patients who started SGLT2i and DPP-4i in the index period, respectively. After propensity score matching, 18583 patients remained in each group and the standardized mean difference decreased substantially for all parameters (online supplemental figure 2, online supplemental table 1). The follow-up times were 635 and 656 days in the SGLT2i 'clean' and the DPP-4i 'clean' cohorts, respectively (online supplemental table 4 ). The distribution of SGLT2i therapies at index date before and after propensity score matching in the SGLT2i 'clean' versus the DPP-4i 'clean' cohort was comparable (online supplemental table 5). Figure 2 shows the Kaplan-Meier survival curves for the individual endpoints. All-cause mortality and the composite endpoint of non-fatal acute myocardial infarction (AMI), stroke and all-cause mortality, HHF, and cancer were associated with lower HR in the SLGT2i 'clean' cohort versus the DPP-4i 'clean' cohort. On the contrary, the risk of LLA was higher in the SGLT2i-treated versus DPP-4i-treated patients. Figure 3 indicates that AMI with PCI (HR, 1.26; 95\% CI 0.97 to 1.64, $\mathrm{p}=0.0771)$ and CT-proven stroke (HR, 0.82; 95\% CI 0.62 to $1.07, \mathrm{p}=0.1421$ ) tended to take different directions. The risk of all-cause mortality was lower by $20 \%$ (HR, 0.80 ; 95\% CI 0.68 to $0.94, \mathrm{p}=0.0057$ ) in the SGLT2i group, but the risk of LLA was $35 \%$ higher (with a wide CI; HR, 1.35 ; $95 \%$ CI 1.03 to $1.77, \mathrm{p}=0.0315$ ) in patients on SGLT2i treatment compared with the DPP-4i-treated cohort (figure 3 ). The hazard of HHF was $19 \%$ lower (HR, $0.81 ; 95 \%$ CI 0.71 to $0.92, \mathrm{p}=0.0018$ ) and the risk of cancer was also $25 \%$ lower (HR, $0.75 ; 95 \%$ CI 0.66 to $0.86, \mathrm{p}<0.0001$ ) in the SGLT2i-treated cohort (figure 3 ). In the case of significantly higher (as in the case of LLA) or lower HR, the number needed to harm $(\mathrm{NNH})$ or the number needed to treat (NNT) values were calculated, respectively (online supplemental table 8 ). At 36 months of follow-up, NNT for all-cause mortality was 116, while that for HHF and cancer were 117 and 94, respectively. Concerning LLA, the NNH value was 313 (online supplemental table 8 ). The rate of secondary prevention was $14.38 \%$ in the SGLT2i 'clean' group versus $14.68 \%$ in the DPP-4i 'clean' group, having either AMI with PCI, coronary artery bypass grafting (CABG), PCI with stenting, stroke, HHF, or LLA in the history (not shown in figures or tables).

In the second analysis, 7167 patients were found in whom SGLT2i was added to DPP-4i, while DPP-4i was switched to SGLT2i in 4891 patients. Propensity score matching resulted in 4843 patients in both arms of investigation (online supplemental figure 3, online supplemental table 2). The mean follow-up times were 657 and 733 days in the SGLT2i 'add-on' and SGLT2i 'switch' cohorts, respectively (online supplemental table 6). The distribution of SGLT2i therapies at index date before and after propensity score matching in the SGLT2i 'add-on' versus the SGLT2i 'switch' cohort was comparable (online supplemental table 7). Figure 4 shows the Kaplan-Meier survival curves for the individual endpoints. All-cause mortality and the composite endpoint of non-fatal AMI, stroke and all-cause mortality were lower in the SGLT2i 'add-on' versus the SGLT2i 'switch' group; however, the risk of AMI, stroke, HHF and cancer was not different between the groups. Additionally, the rate of LLA was not different between the SGLT2i 'add-on' versus the SGLT2i 'switch' cohort. The all-cause mortality curves of the SGLT2i 'add-on' and that of the SGLT2i 'switch' cohort started to diverge from approximately the eighth month on. In figure 5 the risk of AMI, stroke, LLA, HHF and cancer did not show any 


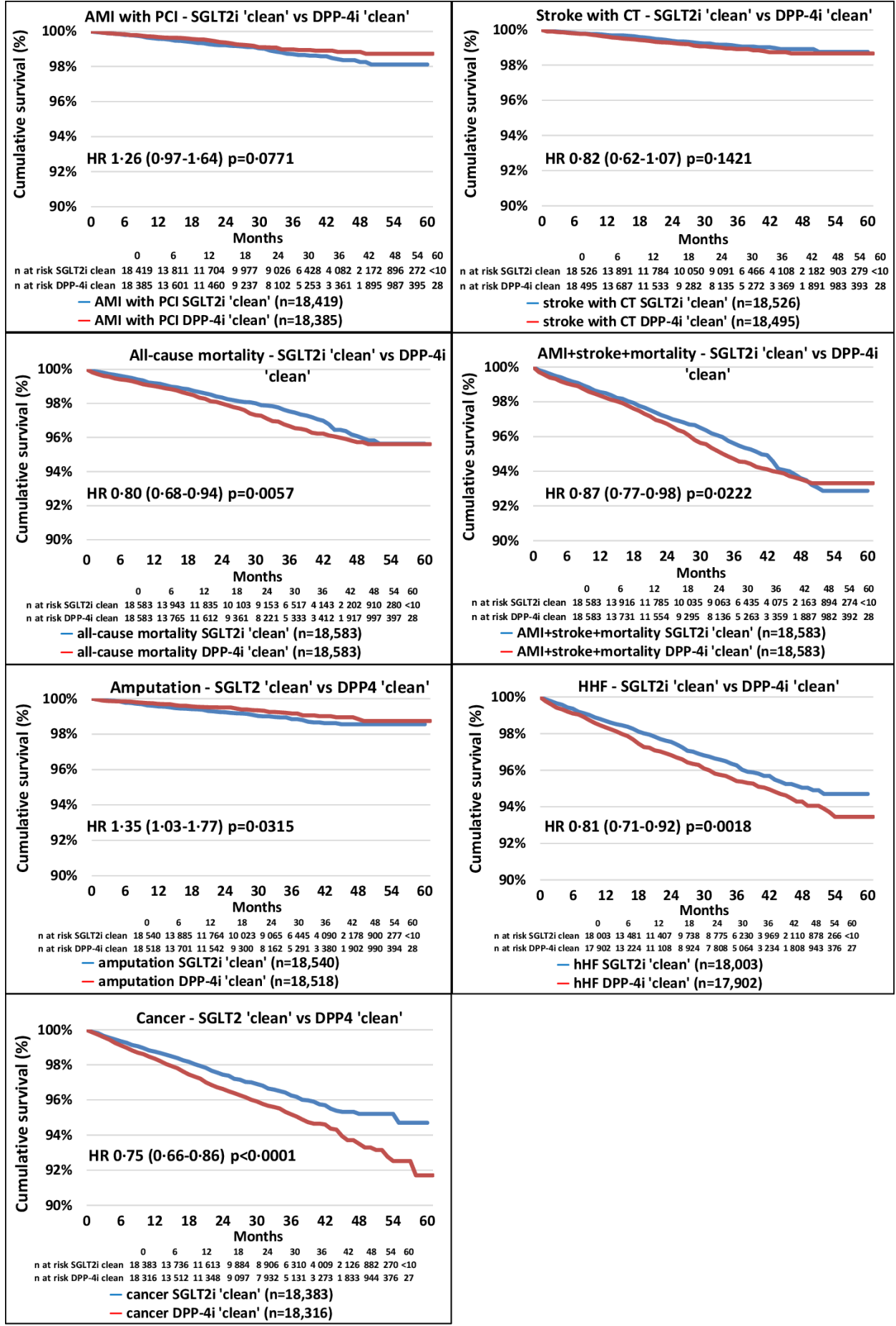

Figure 2 Kaplan-Meier survival curves of major complications and mortality in the propensity score-matched SGLT2i 'clean' versus DPP-4i 'clean' cohorts. The red lines represent the DPP-4i 'clean' cohort, while the blue lines represent the SGLT2i 'clean' cohort. The number of patients at risk is different in the individual outcomes because patients were censored if they had that particular outcome in the previous 1 year. AMI, acute myocardial infarction; DPP-4i, dipeptidyl peptidase-4 inhibitor; HHF, hospitalization for heart failure; PCI, percutaneous coronary intervention; SGLT2i, sodium-glucose cotransporter-2 inhibitor.

significant difference between the two cohorts. However, all of these parameters except for AMI+PCI tended to be lower in the SGLT2i 'add-on' cohort in comparison with the SGLT2i 'switch' group (figure 5). All-cause mortality was $54 \%$ lower (HR, 0.46 ; $95 \%$ CI 0.31 to 0.67 , $\mathrm{p}=0.0001)$. Since there was a highly significant difference in mortality favoring SGLT2i 'add-on' treatment,
NNT was calculated (online supplemental table 8). At the end of the third year this amounted to 57 . The prevalence of secondary prevention in the SGLT2i and DPP-4i 'add-on' group was $13.52 \%$ vs $13.28 \%$ in the SGLT2i 'switch' group, having either AMI with PCI, CABG, PCI with stenting, stroke, HHF, or LLA in the history (not shown in figures or tables). 


\section{Population (n) Events (n) \\ SGLT2i vs DPP-4i SGLT2i vs DPP-4i}

AMI with $\mathrm{PCl}$

Stroke with CT

18,419 vs 18,385

135 vs 99

All-cause mortality

18,526 vs 18,495

18,583 vs 18,583

AMI+stroke+mortality 18,583 vs 18,538

Amputation

HHF

Cancer
18,540 vs 18,518

18,003 vs 17,902

18,383 vs 18,316
99 vs 113

287 vs 333

496 vs 532

127 vs 88

410 vs 470

408 vs 506
Hazard ratio p-value

$1.26(0.97-1.64) \quad p=0.0771$

$0.82(0.62-1.07) p=0.1421$

$0.80(0.68-0.94) p=0.0057$

$0.87(0.77-0.98) p=0.0222$

$1.35(1.03-1.77) p=0.0315$

$0.81(0.71-0.92) p=0.0018$

$0.75(0.66-0.86) p<0.0001$

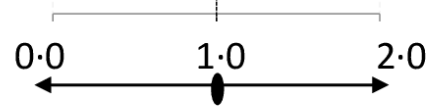

Favors SGLT2i 'clean' cohort

Favors DPP-4i 'clean' cohort

Figure 3 Major complications and mortality in the propensity score-matched SGLT2i 'clean' versus DPP-4i 'clean' cohorts. $P$ values are derived from the Cox proportional hazards model. The number of patients at risk is different in the individual outcomes because patients were censored if they had that particular outcome in the previous 1 year. AMI, acute myocardial infarction; DPP-4i, dipeptidyl peptidase-4 inhibitor; HHF, hospitalization for heart failure; PCl, percutaneous coronary intervention; SGLT2i, sodium-glucose cotransporter-2 inhibitor.

\section{DISCUSSION}

Using the database of the Hungarian NHIF, we obtained data for all patients using SGLT2i and/or DPP-4i between August 2014 and July 2017. All-cause mortality and the major causes of mortality such as CV complications and the risk of cancer were evaluated. First, two propensity score-matched groups were created: one taking SGLT2i but not DPP-4i, and another group where conversely the patients were taking DPP-4i but not SGLT2i ('clean' treatments). The most important findings were that all-cause mortality, the composite endpoint of non-fatal AMI, stroke and all-cause mortality, HHF, and risk of cancer were $20 \%, 13 \%, 19 \%$, and $25 \%$ lower after SGLT2i treatment compared with DPP-4i treatment, respectively. AMI was practically unchanged, but LLA was $35 \%$ higher in the SGLT2i-treated cohort.

In the second approach a switch to SGLT2i from DPP-4i ('switch' group) was compared with those who initiated an SGLT2i with continuation of DPP-4i ('addon' group). In this part the all-cause mortality was 54\% and the composite endpoint of non-fatal AMI, stroke and all-cause mortality was 36\% lower in the SGLT2i 'add-on' group versus the SGLT2i 'switch' group; however, the risk of AMI, stroke, HHF and cancer did not differ. The rate of LLA was also not different between SGLT2i 'add-on' and SGLT2i 'switch' cohorts.

In our study we found a $20 \%$ lower risk of all-cause mortality in the 'clean' groups. SGLT2i decreased mortality up to $37 \%,{ }^{7}$ or resulted in a non-significant mild decrease ${ }^{10}$ in CVOT, which is in line with our results. Although RWE trials are not superior to randomized controlled trials (RCTs) due to lack of randomization, they may strengthen observations on a broader population. CVD-REAL and CVD-REAL Nordic presented significant $48 \%$ and $49 \%$ improvement in all-cause mortality, respectively. ${ }^{20}{ }^{21} \mathrm{~A}$ post-hoc analysis of the latter study has shown that SGLT2i was associated with a $41 \%$ lower mortality than DPP- $4 \mathrm{i}^{2}{ }^{22}$ Both studies have been methodologically criticized $^{19}$ due to the chances of a so-called immortal-time bias and lag-time bias, which were eliminated in our study. DPP-4i themselves did not demonstrate any effect on mortality in CVOTs.

In our study, keeping the DPP-4i when initiating SLGT2i ('add-on') resulted in 54\% lower mortality as compared with those who have stopped taking the DPP-4i after the SGLT2i has been started ('switch' group). This finding highly argues for the combination of SGLT2i and DPP-4i among patients treated with metformin or sulfonylurea. Whether it is due to the intrinsic properties of the two classes of drugs or to a combination therapy in general, similarly to that observed, for example, in the VERIFY study, ${ }^{23}$ is unknown. Although individual outcomes such as stroke, LLA, HHF and cancer were not statistically different between the two cohorts of the second arm, they may all contribute to a better survival in the 'add-on' group. This suggests that the combination applied could have an additive effect.

The efficacy of SGLT2i in reducing CV complications was confirmed in many CVOTs. Patients with AMI and PCI were identified from the national database. We found that the SGLT2i 'clean' group was not associated with a lower hazard in this regard compared with the DPP-4i 'clean' usage. Similarly, no difference was found between the SGLT2i 'add-on' and 'switch' cohorts with regard to AMI. Nevertheless, the risk of non-fatal AMI was not reduced significantly in SGLT2i trials. ${ }^{7810}$ The risk of AMI in the CVD-REAL study was decreased by $22 \%{ }^{24}$; however, the CVD-REAL Nordic study failed to present a lower prevalence of AMI. ${ }^{21}$ CVOTs compared the investigated drug with placebo on top of standard treatment. Consequently, these trial results are not directly comparable with our data; however, the results with regard to AMI are in line with RCT 


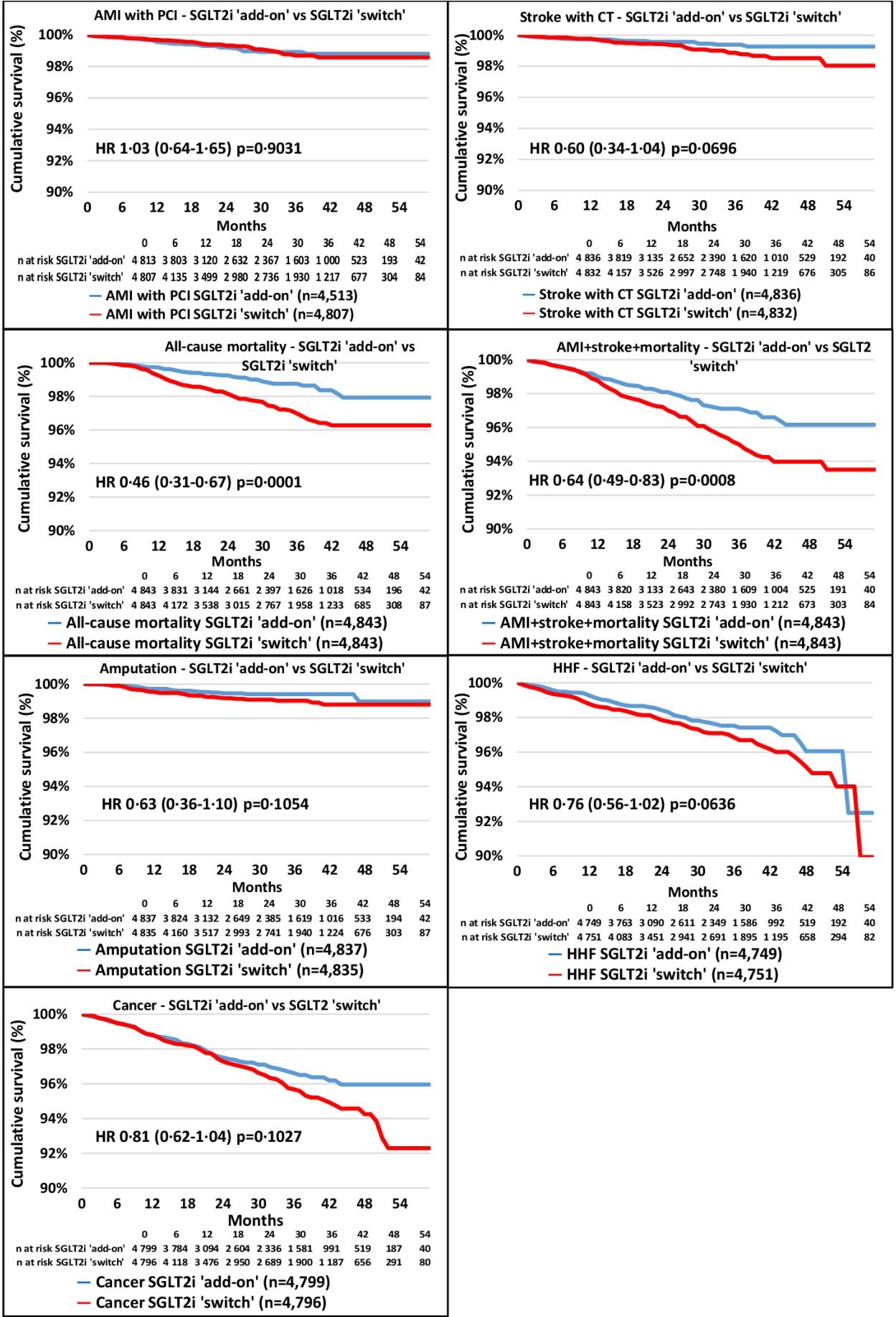

Figure 4 Kaplan-Meier survival curves of major complications and mortality in the propensity score-matched SGLT2i 'addon' versus DPP-4i 'switch' cohorts. The blue lines represent the SGLT2i 'add-on' cohort, while the red lines refer to the SGLT2i 'switch' cohort. The number of patients at risk is different in the individual outcomes because patients were censored if they had that particular outcome in the previous 1 year. AMl, acute myocardial infarction; DPP-4i, dipeptidyl peptidase-4 inhibitor; HHF, hospitalization for heart failure; PCl, percutaneous coronary intervention; SGLT2i, sodium-glucose cotransporter-2 inhibitor.

data and partially with the RWE data on SGLT2i and DPP-4i.

Stroke is another frequent complication of diabetes which leads to long-term disability and may occur substantially more frequently in patients with diabetes than in individuals without diabetes. There was no difference in the prevalence of stroke, neither in the
SGLT2i 'clean' versus the DPP-4i 'clean' cohort nor in the SGLT2i 'add-on' versus 'switch' comparisons. Although the design of previous clinical trials examining the effect of SGLT2i and DPP-4i on stroke does not allow a direct comparison of study results, neither of these drugs exert a substantial effect on the prevalence of stroke in diabetes. ${ }^{24}$ 


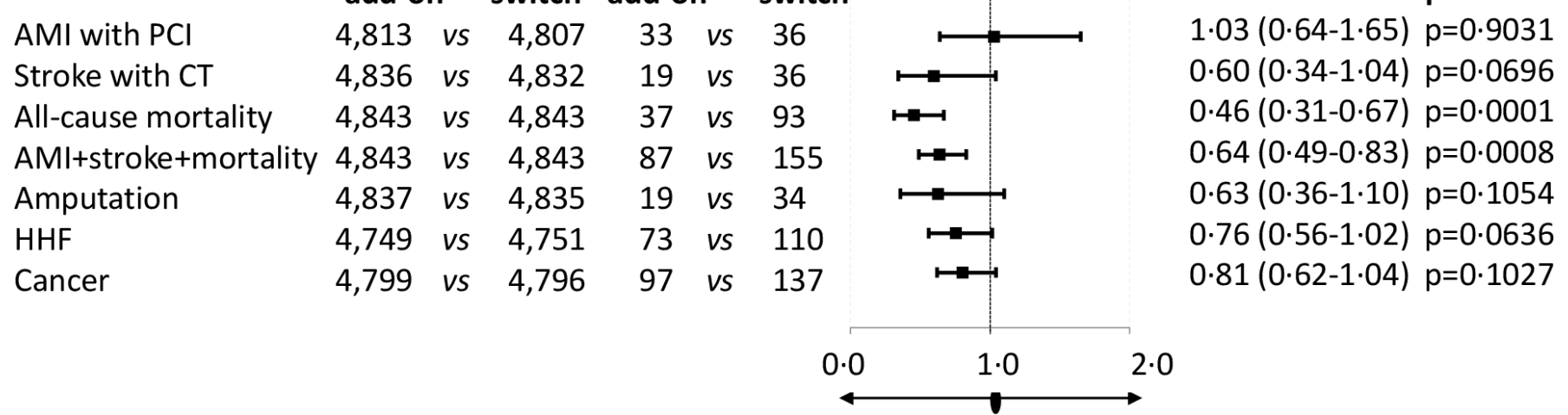

Favors SGLT2i 'add-on' cohort Favors SGLT2i 'switch' cohort Figure 5 Major complications and mortality in the propensity score-matched SGLT2i 'add-on' versus SGLT2i ' switch' cohorts. P values are derived from the Cox proportional hazards model. The number of patients at risk is different in the individual outcomes because patients were censored if they had that particular outcome in the previous 1 year. AMI, acute myocardial infarction; HHF, hospitalization for heart failure; $\mathrm{PCI}$, percutaneous coronary intervention; SGLT2i, sodium-glucose cotransporter-2 inhibitor.

As expected in our study, HHF was $19 \%$ lower in the SGLT2i 'clean' versus the DPP-4i 'clean' group, in line with CVOTs, where SGLT2i decreased HHF by $27 \%-35 \% .^{7810}$ RWE CVD-REAL ${ }^{20}$ and CVD-REAL Nordic $^{21}$ studies also found a substantial decrease in $\mathrm{HHF}$, at $39 \%$ and $30 \%$, respectively. The most relevant data in line with our results came from the EMPRISE study, where a $50 \%$ decrease in HR of HHF was found favoring an SGLT2i compared with a DPP-4i. ${ }^{25}$ There was no significant difference between the SGLT2i 'add-on' and 'switch' groups with regard to HHF. This unequivocal finding suggests a group effect of SGLT2i on HHF.

LLA is a major complication of diabetic angiopathy and neuropathy. Our study has shown that SGLT2i use was associated with a $35 \%$ higher hazard of LLA as compared with DPP-4i. A $132 \%$ increase of hazard for LLA was observed in the Swedish-Danish nationwide registry ${ }^{26}$ in the SGLT2i group compared with the GLP-1 RA group. In the SGLT2i group a $38 \%$ higher risk was detected compared with the DPP-4i population in a big US propensity-matched cohort ${ }^{27}$ study. In this trial noncanagliflozin SGLT2i showed an HR of 2.25 and canagliflozin an HR of 1.15. A 97\% increase was found for LLA after an SGLT2i treatment. ${ }^{10}$ LLA has increased with $25 \%$ in a recent meta-analysis, however with high heterogeneity and mainly due to the results of the CANVAS study. ${ }^{10} 28$ It should be emphasized that canagliflozin is not marketed in Hungary, so the higher rate of LLA in our study cannot be attributed to canagliflozin treatment. On the one hand, the risk of LLA is higher on SGLT2i treatment, while on the other hand the allcause mortality is lower in patients treated with SGLT2i. This finding may suggest that a lower all-cause mortality may save patients for LLA in the SGLT2i arm, since the major determinant of mortality in patients with T2DM is composed of CV diseases and cancer-related death. If mortality decreases in an observational group, the likelihood of an occurrence of a non-fatal event may increase. Cancer-related death was also lower in SGLT2i-treated patients than in those on DDP-4i. The combination of SGLT2i and DPP-4i results in a non-significantly lower risk of LLA of $37 \%$ in comparison with switching from DPP-4i to SGLT2i, although the effect of DPP-4i and SGLT2i on LLA is currently not well understood. These results suggest that combination with DPP-4i may lower the risk of LLA associated with SGLT2i.

Diabetes itself is associated with a substantially higher risk of cancer, with relative risks ranging from 1.2 to 2.5 for different cancer sites except for prostate. ${ }^{16}$ Pancreatic cancer is up to 7.61 times higher than in patients without diabetes. ${ }^{17}$ We have found a $25 \%$ lower hazard of cancer in the SLGT2i 'clean' versus the DPP-4i 'clean' cohort. There are conflicting results on the prevalence of cancer in patients treated with glucose-lowering compounds. Initially, both GLP-1 RA and DPP-4i were suspected to lead to an increased risk of pancreatic and thyroid cancer; however, today, these concerns have been resolved. ${ }^{29}$ Some studies refer to a possible increase in cancer using SGLT2i, mainly bladder cancer, while others suggest a protective effect, for example in case of canagliflozin and gastrointestinal tract cancer. ${ }^{30}$ Incidence of cancer was not reported for empagliflozin ${ }^{7}$ and was not altered by canagliflozin ${ }^{10}$ in CVOTs. The overall rate was not different in dapagliflozin-treated patients, except for a $43 \%$ lower prevalence of bladder cancer. ${ }^{8}$ In our study, the additive use of SGLT2i and DPP-4i (in the SGLT2i 'add-on' group) was not significantly associated with a lower risk of cancer as compared with those patients who stopped taking DPP-4i after initiation of an SGLT2i. The follow-up of patients was in line with or even longer than previous landmark RCT studies in the field of diabetes (SAVORTIMI, 2.1; EXAMINE, 1.5; TECOS, 3; CARMELINA, 2.2; 
EMPA-REG, 3.1; CANVAS, 2.4; DECLARE-TIMI, 4.2; EMPEROR-REDUCED, 1.33; DAPA-HF, 1.5; ELIXA, 2.1; LEADER, 3.8; SUSTAIN-6, 2.1; EXCEL, 3.2; HARMONY, 1.6).$^{31}$ Especially in the case of RWE studies (CVD-REAL, approximately 0.9; CVD-REAL Nordic, 0.95 years $),{ }^{31}$ the follow-up time was short, generally less than 1-1.5 years. The risk of cancer was evaluated as a safety measure in many of these studies. Altogether, the 3-year observational period has already provided data showing a striking difference in mortality between the groups investigated.

The strengths of our study include providing a nationwide approach, since most citizens have received medical coverage from the NHIF; therefore, these data provide a near full coverage of the Hungarian population with diabetes. Also, thorough propensity score matching, including significant CV and non-vascular comorbidities and drug therapy, was performed. We would like to draw special attention to the well-matched usage of $\mathrm{CV}$ drugs as well as heart failure medications. Additionally, because of earlier criticism on RWE data, ${ }^{19}$ we eliminated immortal-time bias and time-lag bias.

The limitations of this study are the lack of laboratory as well as anthropometric data, and the retrospective, nonrandomized nature of the study in line with other RWE studies. There are no $\mathrm{HbA}_{1 \mathrm{c}}$ data available, which may lead to possible confounding effect since patients with SGLT2i added to DPP-4i may associate with more severe diabetic status compared with switchers. However, patients with a suspected more severe condition in the add-on group had better outcomes than those in the switch group, with a potentially milder diabetes. This finding in fact is rather a strength of this study not a limitation. Furthermore, we are also aware that the last modification of the ADA/ EASD joint statement recommends CV protective agents, irrespective of the current or desired $\mathrm{HbA}_{1 \mathrm{c}}$ value. ${ }^{12}$ Renal function, including actual glomerular filtration rate (GFR), was also not available for inclusion in the analysis since the database consists of ICD codes only. Patients on SGLT2i treatment may have had better renal function, as suggested by prescription label that currently allows initiation of SGLT2i at a GFR of $>60 \mathrm{~mL} / \mathrm{min} / 1.73 \mathrm{~m}^{2}$ only. However, risk factors of chronic kidney disease were well balanced between the groups of different treatments. In total 54 parameters were involved in propensity score matching. Only 1 out of 54 parameters has shown a mild imbalance after matching both cohorts. The standardized mean difference (SMD) for the index year in the first cohort was 0.153 and that for metformin use was 0.113 . The reason for incomplete matching may be that the unmatching of these parameters was high before propensity score matching, $58 \%$ for the index year and $11.5 \%$ for metformin treatment. However, the other 53 parameters were properly balanced in each cohort, so the outcome of the survival analysis might not have been influenced.

\section{CONCLUSIONS}

In conclusion, our results represent a nationwide experience on SGLT2i treatment, a lower risk of overall mortality and cancer, a lower chance of developing HHF, and a higher chance of LLA, as compared with DPP4i. Furthermore, adding SGLT2i to DPP-4i instead of switching from DPP-4i to SGLT2i may prove to be a beneficial approach in terms of further improving all-cause mortality and neutralizing the effect of SGLT2i on the risk of LLA.

\section{Author affiliations}

${ }^{1}$ Second Department of Medicine and Nephrology-Diabetes Centre, University of Pécs Medical School, Pécs, Hungary

${ }^{2}$ RxTarget Ltd, Szolnok, Hungary

${ }^{3}$ Department of Rheumatology, Faculty of Medicine, University of Debrecen, Debrecen, Hungary

${ }^{4}$ National Institute of Rheumatology and Physiotherapy, Budapest, Hungary ${ }^{5}$ Bajcsy-Zsilinszky Hospital, Budapest, Hungary

${ }^{6}$ First Department of Medicine, Faculty of Medicine, Semmelweis University, Budapest, Hungary

Acknowledgements The authors would like to thank colleagues at the National Institute of Health Insurance Fund for their help in providing the data for the study and would also like to thank Professor Dr József Andor for linguistic help and Enikö Bodor for technical assistance.

Contributors IW, GJ, PK, GP, and ZS provided the idea for the study and were involved in the interpretation of data and discussion of the results. GS and GAM interpreted the data and wrote the paper with input from all authors. GR, IF, and ZK obtained, analyzed, and interpreted the data and wrote the paper with input from all authors. All authors provided critical feedback and contributed to the research, analysis, and preparation of the manuscript.

Funding The Hungarian Diabetes Association and the Hungarian Society of Rheumatology provided funding for data collection and research.

\section{Competing interests None declared.}

\section{Patient consent for publication Not required.}

Ethics approval The study protocol was approved by the Ethical Board of the University of Pécs (10338-5/2019/EKU) and by NHIF (study license number: 1043/122/2019).

Provenance and peer review Not commissioned; externally peer reviewed.

Data availability statement All data relevant to the study are included in the article or uploaded as supplemental information. The study was performed using the National Institute of Health Insurance Fund database. The database is not publicly available and the requested data were released upon ethical committee approval.

Supplemental material This content has been supplied by the author(s). It has not been vetted by BMJ Publishing Group Limited (BMJ) and may not have been peer-reviewed. Any opinions or recommendations discussed are solely those of the author(s) and are not endorsed by BMJ. BMJ disclaims all liability and responsibility arising from any reliance placed on the content. Where the content includes any translated material, BMJ does not warrant the accuracy and reliability of the translations (including but not limited to local regulations, clinical guidelines, terminology, drug names and drug dosages), and is not responsible for any error and/or omissions arising from translation and adaptation or otherwise.

Open access This is an open access article distributed in accordance with the Creative Commons Attribution Non Commercial (CC BY-NC 4.0) license, which permits others to distribute, remix, adapt, build upon this work non-commercially, and license their derivative works on different terms, provided the original work is properly cited, appropriate credit is given, any changes made indicated, and the use is non-commercial. See: http://creativecommons.org/licenses/by-nc/4.0/.

ORCID IDs

Gergő A Molnár http://orcid.org/0000-0001-6052-5907

István Wittmann http://orcid.org/0000-0001-5163-5733

\section{REFERENCES}

1 Kiss Z, Rokszin G, Abonyi-Tóth Z, et al. Different changes of risks for stroke and myocardial infarction in patients with type 2 diabetes 
in Hungary between the two periods of 2001-2004 and 2010-2013. Front Endocrinol 2019;10:1-5.

2 Kiss Z, Rokszin G, Abonyi-Tóth Z, et al. Young adult patients with type 1 diabetes have a higher risk of mortality than those of similar age with type 2 diabetes: a nationwide analysis in Hungary. Diabetes Metab Res Rev 2019;35:1-7.

3 Kiss Z, Rokszin G, Abonyi-Tóth Z, et al. Dissimilar impact of type 2 diabetes on cardiovascular outcomes according to age categories: a nationwide population study from Hungary. Cardiovasc Diabetol 2018;17:1-8.

4 Raghavan S, Vassy JL, Ho Yuk-Lam, et al. Diabetes mellitus-related all-cause and cardiovascular mortality in a national cohort of adults. $J$ Am Heart Assoc 2019;8.

5 Nwaneri C, Cooper H, Bowen-Jones D. Mortality in type 2 diabetes mellitus: magnitude of the evidence from a systematic review and meta-analysis. Br J Diabetes Vasc Dis 2013;13:192-207.

$6 \mathrm{Xu} \mathrm{G}$, You D, Wong L, et al. Risk of all-cause and CHD mortality in women versus men with type 2 diabetes: a systematic review and meta-analysis. Eur J Endocrinol 2019;180:243-55.

7 Zinman B, Wanner C, Lachin JM, et al. Empagliflozin, cardiovascular outcomes, and mortality in type 2 diabetes. $N$ Engl $\mathrm{J}$ Med 2015;373:2117-28.

8 Wiviott SD, Raz I, Bonaca MP, et al. Dapagliflozin and cardiovascular outcomes in type 2 diabetes. N Engl J Med 2019;380:347-57.

9 Marso SP, Daniels GH, Brown-Frandsen K, et al. Liraglutide and cardiovascular outcomes in type 2 diabetes. N Engl J Med 2016;375:311-22.

10 Neal B, Perkovic V, Mahaffey KW, et al. Canagliflozin and cardiovascular and renal events in type 2 diabetes. N Engl J Med 2017;377:644-57.

11 Shao S-C, Lin Y-H, Chang K-C, et al. Sodium glucose co-transporter 2 inhibitors and cardiovascular event protections: how applicable are clinical trials and observational studies to real-world patients? BMJ Open Diabetes Res Care 2019;7:e000742.

12 Buse JB, Wexler DJ, Tsapas A. Update to: management of hyperglycaemia in type 2 diabetes, 2018. A consensus report by the American diabetes association (ADA) and the European association for the study of diabetes (EASD). Diabetologia 2019;2020:221-8

13 Dicembrini I, Tomberli B, Nreu B, et al. Peripheral artery disease and amputations with sodium-glucose co-Transporter-2 (SGLT-2) inhibitors: a meta-analysis of randomized controlled trials. Diabetes Res Clin Pract 2019;153:138-44.

14 Arnott C, Li Q, Kang A, et al. Sodium-Glucose cotransporter 2 inhibition for the prevention of cardiovascular events in patients with type 2 diabetes mellitus: a systematic review and meta-analysis. $J$ Am Heart Assoc 2020;9:e014908.

15 Rådholm K, Zhou Z, Clemens K, et al. Effects of sodium-glucose co-transporter-2 inhibitors in type 2 diabetes in women versus men. Diabetes Obes Metab 2020;22:263-6.

16 Cohen DH, LeRoith D. Obesity, type 2 diabetes, and cancer: the insulin and IGF connection. Endocr Relat Cancer 2012;19:F27-45.

17 Cui Y, Andersen DK. Diabetes and pancreatic cancer. Endocr Relat Cancer 2012;19:F9-26.
18 Jermendy G, Kempler P, Abonyi-Tóth Z, et al. Changes in features of diabetes care in Hungary in the period of years 2001-2014. Orv Hetil 2016;157:1259-65.

19 Suissa S. Lower risk of death with SGLT2 inhibitors in observational studies: real or bias? Diabetes Care 2018;41:6-10.

20 Kosiborod M, Cavender MA, Fu AZ, et al. Lower risk of heart failure and death in patients initiated on sodium-glucose cotransporter-2 inhibitors versus other glucose-lowering drugs: the CVD-REAL study (comparative effectiveness of cardiovascular outcomes in new users of sodium-glucose cotransporter-2 inhibitors). Circulation 2017;136:249-59.

21 Birkeland KI, Jørgensen ME, Carstensen B, et al. Cardiovascular mortality and morbidity in patients with type 2 diabetes following initiation of sodium-glucose co-transporter-2 inhibitors versus other glucose-lowering drugs (CVD-REAL Nordic): a multinational observational analysis. Lancet Diabetes Endocrinol 2017;5:709-17.

22 Persson F, Nyström T, Jørgensen ME, et al. Dapagliflozin is associated with lower risk of cardiovascular events and all-cause mortality in people with type 2 diabetes (CVD-REAL Nordic) when compared with dipeptidyl peptidase-4 inhibitor therapy: A multinational observational study. Diabetes Obes Metab 2018;20:344-51.

23 Matthews DR, Paldánius PM, Proot $\mathrm{P}$, et al. Glycaemic durability of an early combination therapy with vildagliptin and metformin versus sequential metformin monotherapy in newly diagnosed type 2 diabetes (verify): a 5-year, multicentre, randomised, double-blind trial. Lancet 2019;394:1519-29.

24 Kosiborod M, Birkeland KI, Cavender MA, et al. Rates of myocardial infarction and stroke in patients initiating treatment with SGLT2inhibitors versus other glucose-lowering agents in real-world clinical practice: results from the CVD-REAL study. Diabetes Obes Metab 2018;20:1983-7.

25 Patorno E, Pawar A, Franklin JM. Empagliflozin and the risk of heart failure hospitalization in routine clinical care: a first analysis from the EMPRISE study. Circulation 2019;139:2822-30.

26 Ueda P, Svanström H, Melbye M, et al. Sodium glucose cotransporter 2 inhibitors and risk of serious adverse events: nationwide register based cohort study. BMJ 2018;363:k4365.

27 Adimadhyam S, Lee TA, Calip GS, et al. Risk of amputations associated with SGLT2 inhibitors compared to DPP-4 inhibitors: a propensity-matched cohort study. Diabetes Obes Metab 2018:20:2792-9.

28 Zelniker TA, Wiviott SD, Raz I, et al. SGLT2 inhibitors for primary and secondary prevention of cardiovascular and renal outcomes in type 2 diabetes: a systematic review and meta-analysis of cardiovascular outcome trials. Lancet 2019;393:31-9.

29 Davies MJ, D'Alessio DA, Fradkin J, et al. Management of hyperglycaemia in type 2 diabetes, 2018. A consensus report by the American diabetes association (ADA) and the European association for the study of diabetes (EASD). Diabetologia 2018:61:2461-98.

30 Abouelkheir M, Taha AE. SGLT2 inhibitors and cancer: is immunity the missing link? J of Pharmacol \& Clin Res 2019;6:555699.

31 Zou C-Y, Liu X-K, Sang Y-Q, et al. Effects of SGLT2 inhibitors on cardiovascular outcomes and mortality in type 2 diabetes. Medicine 2019;98:e18245 